\title{
High Throughput Sample Preparation using Ultrafiltration in Multiwell Plate Format : Post-Sequencing Cleanup of Dye Terminator Sequencing Reactions for Capillary Electrophoresis
}

\author{
Ali Chaudry *, Dan Collins *, Duane Romana *, Cathryn Crego *, \\ Peter Hewitt *, Haiying Wang *, Rohini Dhulipala *, Scott Duthie *, \\ Martin Smith ${ }^{* *}$, Jonathan Lipsky ${ }^{* *}$, Johan Wahlberg*, \\ and James C. Davis
}

*Amersham Pharmacia Biotech Inc., 800 Centennial Avenue, P.O. Box 1327, Piscataway, NJ, USA

${ }^{* *}$ Whatman, Inc., 136 Weymouth Street, Rockland, MA 02370, USA

${ }^{* * *}$ Person to whom correspondence should be addressed

\begin{abstract}
A unique new method is described showing the use of microfiltration and ultrafiltration membranes in conjunction with gel permeation in 96 well plates. Using this technique it is possible to clean up DNA sequencing reactions to a level of template DNA purity that makes high DNA loading onto capillary electrophoresis (CE) sequencers possible. Loadings as high as $10,000 \mathrm{ng}$ allow sequencing on $\mathrm{CE}$ to be equal in performance to gel slab electrophoresis. This technique replaces alcohol precipitation for sequencing reaction cleanup with an easily automated process taking $1 / 6$ the time.
\end{abstract}

Key words : DNA sequencing / dye terminator removal / ultrafiltration

\section{INTRODUCTION}

Multiwell plates equipped with coarse, microfiltration and specialty membranes are well known and have been employed for high throughput, automated sample preparation for many years ${ }^{1)}$. The choice of polymeric microporous membranes for encapsulation within such multiwell devices is therefore extremely important when considering characteristics such as flow rate, pore size, chemical resistance, and non-specific binding. Of particular interest is non-specific binding due to the enormous surface area present in (UF) and microfiltration (MF) membranes. Such binding can affect recovery and performance of the devices in many biological and biochemical separations. For high value or rare products, product recovery (or loss due to binding) can be both a scientific and financial issue. As higher purity, lower concentration and higher value biotechnology products are frequently processed, the issue of non-specific binding or fouling of the membrane becomes ever more critical to the 
researcher. Specifically engineered membranes from surface and bulk modified polyethersulfones can be utilized to prevent, or at least minimize, non-specific binding ${ }^{2)}$. Further, polysulfone type polymers have been used to manufacture UF membranes which have been utilized for desalting, sample concentration, deproteinization, and buffer exchange $\mathrm{e}^{3 \text {. }}$.

The incorporation of ultrafiltration membranes into multiwell devices, particularly 96 well and higher, is difficult due to the nature of the membrane and the humectants required to prevent pore collapse. It is unreasonable to expect that 96 filter disks can be inserted individually into the wells and tightened to leak-free tolerances. However, products such as the Whatman, Inc. 96 well SPX line are produced with technology allowing simultaneous inserting and sealing of the filter, thus allowing commercial 96 well UF devices for the first time. These plates are provided with a range of UF membranes ranging in molecular weight cut-off from 10,000 to 300,000 Daltons. They are typically provided in a 96 well polystyrene plate with $350 \mu l$ volume per well. The plates are equipped with long drip directors on the base for cross-talk free collection of the ultrafiltrate. Transmembrane pressure is typically provided by centrifuging the plate at 500 to $1,000 \mathrm{~g}$, although slow filtration is possible on the high cut-off membrane through the use of vacuum for filtration.

Although there are a myriad of applications for UF in multiwell devices, this paper will describe a particular application of critical importance to the rapidly growing science of DNA purification, amplification, and sequencing.

The development of capillary electrophoresis (CE) technology for DNA sequencing ${ }^{4)}$ has proven to be both efficient and accurate for large-scale genome sequence determination. In comparison to slab gel electrophoretic methods, the combination of $\mathrm{CE}$ with fluorescent labeled ddNTPs for detection has significantly shortened the turnaround time to results. CE technology has also resulted in the development of electrokinetic injection (sample loading) methods. The efficiency of electrokinetic injection is dependent upon the applied voltage and sample ion composition. Consequently, electrokinetic injection methods require more stringent cleanup of samples to remove excess salts and unincorporated fluorescent ddNTPs.

The common method for pre-CE cleanup is ethanol precipitation followed by re-suspension of the DNA pellet. This paper will describe an alternative method using a multiwell plate containing a UF membrane. A direct comparison is made using an identical multiwell plate but containing a 0.45 micron PVDF membrane, instead.

\section{METHOD}

Studies with 96 well plates using MF membrane downstream from size-exclusion gel were done using AutoSeq96 plates, available from Amersham Pharmacia Biotech.

All 96 well UF plates used were 100,000 molecular weight cutoff polysulfone available from Whatman, Inc. Each well in the plates was subsequently filled with $50 \mu \mathrm{g}$ of size-exclusion gel. The plates were centrifuged at $910 \times \mathrm{g}$ for 5 minutes to remove the rehydration medium from the gel. A $15 \mu l$ volume of sample from the sequencing reaction mixture was added to each well and recovered at high purity in a 96 well collection plate by centrifugation at $910 \times \mathrm{g}$ for 5 minutes, unless otherwise specified.

Sequencing reaction were performed as outlined in the DYEnamic ET dye terminator kit (MegaBACE), Amersham Pharmacia Biotech, 


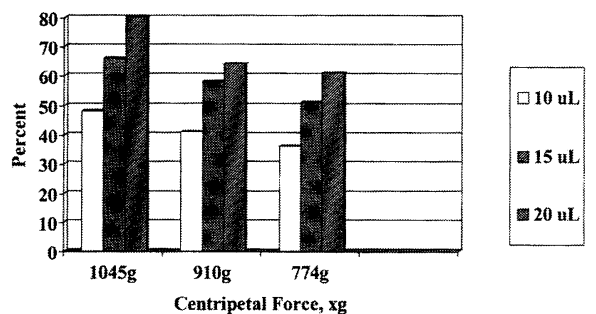

Fig. 1 Comparison of centripetal force and loading volume with percent recovery of Sanger fragments

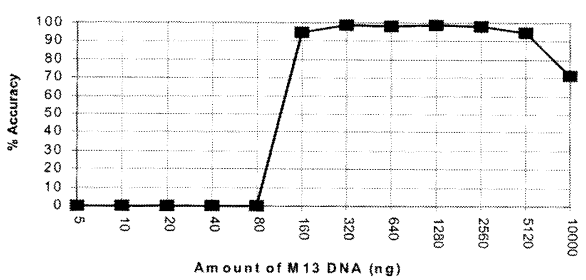

Fig. 3A Accuracy of generated sequence at 500 bases with increasing amounts of template DNA

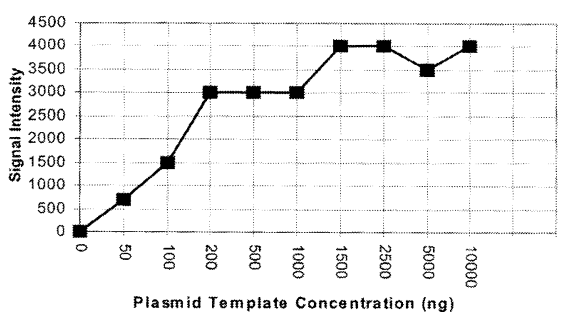

Fig. 3B Ultrafiltration Technology, Average signal intensity using increasing amounts of template DNA in the sequencing reactions

Inc. \#US81090. All sequences were obtained using an Amersham Pharmacia MegaBACE Sequencer.

\section{RESULTS}

Read length is an empirical determination of the accuracy of a DNA sequence determination over a several hundred base fragment. A read length of at least 500 at $98.5 \%$ base call accuracy is highly desirable to the DNA sequencing community. The base call accuracy is the proba-

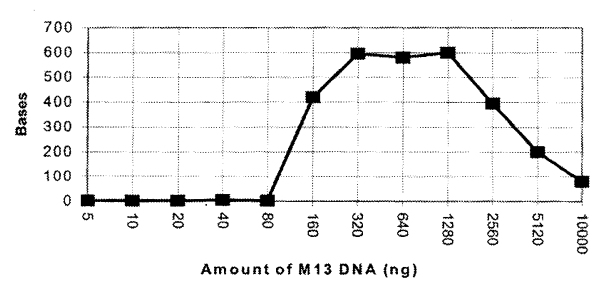

Fig. 2A Number of Bases at $98.5 \%$ accuracy with increasing amounts of template DNA

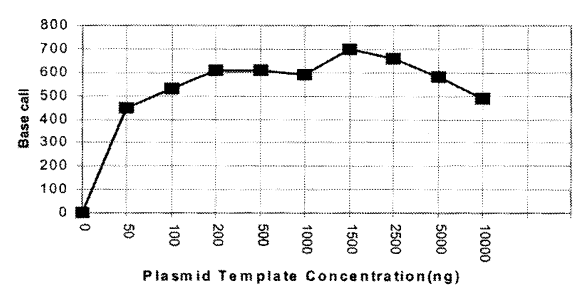

Fig. 2B Ultrafiltration Technology, Average Bases Calls at $98.5 \%$ Accuracy

bility of the instrument identifying the base (either A, G, T or C) correctly. Accuracy typically deteriorates the further out the DNA fragment the instrument is reading. The more pure the sample, the larger the amount of material of interest that can be applied to the instrument. Thus, a product with purity that will allow $10 \mu \mathrm{g}$ of DNA to be loaded can provide higher accuracy.

Fig. 1 shows the results of experiments to maximize the purity and recovery of DNA fragments using different loadings and $g$-force on the multiwell plate. The highest recovery was achieved at the highest $\mathrm{g}$-force, $1040 \times \mathrm{g}$. However, overall purity and recovery peaked at $910 \times \mathrm{g}$ and those conditions were used for all subsequent experiments.

Fig. 2 compares the total number of bases read (read length) versus amount of sample applied for two series of experiments. Fig. 2A illustrates the read results with the MF membrane. Note that sample size applied to obtain the desirable $>500$ base read length at $98.5 \%$ 
base call accuracy spans a range of 200 to 2,200 ng of DNA sample applied. In comparison, Fig. $2 \mathrm{~B}$ shows the results from identical experiments with the UF membrane. Clearly, the UF membrane provides a much greater range of sample of template DNA than the MF product. The "acceptable" range of sample application is a low as $90 \mathrm{ng}$ and a high as 10,000 ng. Since the molecular biologist can never be certain how much sample he will obtain from the PCR and sequencing reactions, a greater range of usable sample quantity is essential.

Due the presence of BAC, Taq, or other impurities, accuracy of base calls can deteriorate seriously with low sample volumes. This is principally due to decrease in signal intensity or signal to noise ratio. Fig. $3 \mathrm{~A}$ exemplifies the results from a series of experiments using an MF membrane in the multiwell plate where the amount of template DNA was increased from 5 $\mathrm{ng}$ to $10,000 \mathrm{ng}$. The chart shows the same acceptable range of sample quantity for $98.5 \%$ accuracy that was shown in Fig. 2A. Note that virtually no accuracy was possible below $100 \mathrm{ng}$. Fig. 3B, for experiments using a multiwell plate with the UF membrane, shows the nearly linear response of signal intensity to increasing amounts of template DNA in the sample. Good base read accuracy is possible over a much wider range of sample size than with the MF membrane. This correlates well with the data in Fig. 2B showing acceptable read accuracy over a range of sample size from $90 \mathrm{ng}$ to $10,000 \mathrm{ng}$.

\section{DISCUSSION}

Due to the complex mixture of components in these types of samples, it is difficult to clearly determine what the UF and MF membranes are actually accomplishing. Coupling that with the fact that these plates are really hybrid separations systems utilizing both size exclusion and

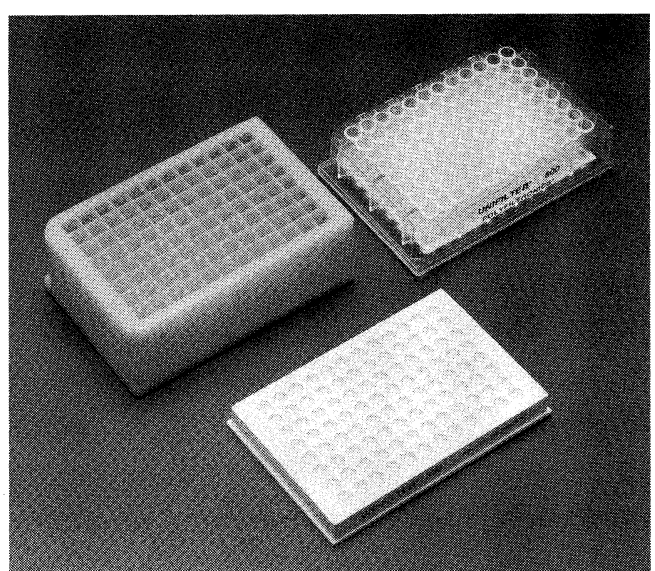

Fig. 4 UniFilter ${ }^{\circledR}$ (Filter Bottm Microplates)

filtration, we can come to some reasonable grasp of the overall processes, however.

In the typical mixture from a PCR reaction, there can be buffers, salts, proteins, bases, and naturally, ssDNA segments. From a sequencing reaction, the components include salts, florescent indicators (ddNTPs), and other relatively low molecular weight components. In addition, there can be proteins, BAC (bacterial artificial chromosome), Taq and other DNA related high molecular weight components. BACs and Taqs are very large molecules with up to 200 kilobases in the loaded form. In this entire mixture reside the DNA fragments of interest-typically less than a kilobase. Consequently, they reside in the middle of the range of molecular weight components in the mixture.

When the sample passes through the size exclusion media, most of the undesirable low molecular weight components are removed while the DNA templates, the proteins and the BAC or Taq are allowed to pass around the gel particles. When the MF membrane is downstream of the gel, all of these components pass into the sample collection plate. However, with the UF membrane downstream of the gel, much of the very high molecular weight materials are removed, thus providing the enhancement in 
template DNA sample purity and consequently far better results from $\mathrm{CE}$ sequencing.

\section{CONCLUSIONS}

Using the AutoSeq96 multiwell plates allowed reproducibility in desalting and dye removal with significant time savings over conventional alcohol precipitation methods. Only 15 minutes was required for the multiwell plates while a typical alcohol precipitation takes 90 minutes. The process is stable at ambient temperature, a desirable factor for high through-put automation. In addition, sequencing reaction template sample size could be increased up to $2,500 \mathrm{ng}$, significantly higher than the levels possible without purification of the sequencing reaction mixture.

Addition of a UF membrane downstream of the gel resulted in similar reproducibility in dye and salt removal, time savings, and stability. However, the sample quantity was increased to $10,000 \mathrm{ng}$. That is particularly important since it allows CE to process samples sizes which heretofore could only be processed using slab gel electrophoresis, an ungainly and time consuming alternative to $\mathrm{CE}$.

Note : Amersham Pharmacia Biotech has applied for patents covering the application of gel/MF and gel/UF multiwell plates for sequence reaction cleanup. Whatman Inc. holds and has applied for patents covering the design and composition of multiwell plates containing MU and UF membranes.

\section{References}

1) Burbaum, J : Engines of Recovery, Chemistry in Britain (1998)

2) Pitt, A : The non-specific Protein Binding of Polymeric Microporous Membranes, J. Parenteral Sci. \& Techn.,41, 3, 110-113 (1987)

3) Oroskar, A. : developmental Applications for Membrane-bottom Microwell Plates. IVD Technology, January/February, 40-47 (1998)

4) Ruiz-Martinez, M.C., et al. : Anal. Chem, 70, 1516-1527

（受付 2000 年 4 月 21 日 揭載決定 5 月 22 日) 